\title{
The Current Status of Microvascular Decompression for the Treatment of Hemifacial Spasm in Japan: An Analysis of 2907 Patients Using the Japanese Diagnosis Procedure Combination Database
}

\author{
Yoshifumi Mizobuchi, ${ }^{1}$ Keiji Muramatsu, ${ }^{2}$ Makoto OHTAni, ${ }^{3}$ Junichiro SatOmi, ${ }^{1}$ \\ Kiyohide FushIMI, ${ }^{4}$ Shinya MATSUDA, ${ }^{2}$ and Shinji NAGAHIRO ${ }^{1}$ \\ ${ }^{1}$ Department of Neurosurgery, Institute of Health Biosciences, \\ The University of Tokushima Graduate School, Tokushima, Tokushima, Japan; \\ ${ }^{2}$ Department of Preventive Medicine and Community Health, School of Medicine, \\ University of Occupational and Environmental Health, Kitakyushu, Fukuoka, Japan; \\ ${ }^{3}$ Data Science Center of Occupational Health, \\ University of Occupational and Environmental Health, Kitakyushu, Fukuoka, Japan; \\ ${ }^{4}$ Department of Health Policy and Informatics, Tokyo Medical and \\ Dental University Graduate School, Tokyo, Japan
}

\begin{abstract}
Microvascular decompression (MVD) is widely used as a safe and effective treatment for hemifacial spasm (HFS). However, the extent of application of this therapeutic method and its outcomes in Japan are currently unclear. To address these questions, we analyzed the utilization of MVD for the treatment of HFS during the 33- month period from July 2010 to March 2013. We conducted an analysis on data contained in the Diagnosis Procedure Combination database in Japan. This analysis showed that MVD was used for the treatment of HFS in 2907 cases (men, 916; women, 1991) with 2.2 times more women treated than men. MVD for HFS was most frequently performed in women aged 50 to 69 years; however, most men were aged between 40 and 59 years at the time of the procedure. The numbers of procedures performed per 100,000 population/year were 0.83 overall in Japan, with the numbers larger in prefectures with larger populations. Regarding discharge outcomes, the mortality rate was $0.1 \%$. The mean length of hospital stay in patients undergoing MVD for HFS was 14.7 days. This analysis provides preliminary information regarding the trends in the performance of MVD for the treatment of HFS in Japan. Further studies on other registries that contain data obtained by standardized assessment methods and that include long-term outcomes and postoperative complications are required.
\end{abstract}

Key words: hemifacial spasm, microvascular decompression, Diagnosis Procedure Combination

\section{Introduction}

Hemifacial spasm (HFS) is generally recognized as resulting from compression of the facial nerve, most likely at the nerve root exit zone. ${ }^{1-3)}$ Microvascular decompression (MVD) of the facial nerve root exit zone for the treatment of HFS was initially proposed by Gardner and Sava ${ }^{4)}$ and was subsequently popularized by Jannetta following the introduction of the operative microscope in the 1970s. ${ }^{5,6)}$ MVD has been adopted as a therapeutic method for the treatment of HFS in Japan since that time and is recognized as a safe and definitive treatment for

Received September 18, 2016; Accepted November 7, 2016
HFS. MVD has a proven long-term efficacy when performed at specialized institutes ${ }^{7)}$ and offers significant advantages over alternative treatments, such as neurotoxin (Botox ${ }^{\circledR}$ ) therapy, that provide only a temporary benefit. ${ }^{8,9)}$

However, there have been no studies of the present situation of MVD for HFS in Japan, and the details are unclear. The health care system of Japan has severe financial problems because of the expenses associated with new medical technology, a rapidly aging society, and extended hospitalization periods of patients. ${ }^{10-14)}$ To address these issues, the Ministry of Health, Labour and Welfare (MHLW) and its affiliated research institute have begun investigating whether the Japanese case-mix classification system, namely 
the Diagnosis Procedure Combination (DPC) database, can be used to standardize medical profiling and payment. ${ }^{10-14)}$ Japanese case-mix projects based on the DPC system were introduced to 82 academic hospitals (the National Cancer Center, the National Cardiovascular Center, and 80 university hospitals) in 2003. ${ }^{10-14)}$ Reimbursement from health insurance companies using the DPC system is common practice in Japan. According to the administrative database of the DPC system, the number of acute care hospitals has increased. Enormous amounts of inpatient data are collected annually, covering approximately $90 \%$ of the total acute care inpatient hospitalizations. ${ }^{10-14)}$ Therefore, the data of the DPC system clearly reflect the circumstances of medical care in Japan at the population level. This system collects important data during hospitalization in addition to the characteristics of the unique reimbursement system. Each patient's financial data, claim information, and discharge summary (including the principal diagnosis, complications, and comorbidities during hospitalization) are thoroughly recorded in the DPC administrative database. These data are coded using the International Classification of Diseases and Injuries, 10th Revision (ICD-10) code. Additionally, this administrative database contains comprehensive medical information, including all interventional or surgical procedures, medications, and devices that have been indexed in the original Japanese code. To optimize the accuracy of the recorded diagnoses, physicians are obligated to record the diagnoses based on the patient's medical records.

The purpose of this retrospective study was to analyze data from this Japanese national administrative database to investigate the present situation of MVD for the treatment of HFS in Japan.

\section{Methods}

\section{Data source}

MHLW has stared the Japanese original case-mix program, so called DPC project since 2003. Under the scheme MHLW gathers the DPC related data from all participating institutions (1,863 hospitals in 2014). This database is composed of clinical data as well as claims data for the date, charge, and quantity of medical care items used. Data from hospitals are gathered and merged into a standardized electronic format defined by MHLW. Although this database is useful for various kinds of medical researches, MHLW does not permit its use for such purposes.

Besides the MHLW's DPC project, the DPC Research Institute gathers the DPC related data from the contracted hospitals, in order to facilitate the DPC base health research. In this study, we used the inpatient data for the 33-month period from July 1, 2010, to March 31, 2013. All of the institutions and hospitals that provided detailed data granted permission for the use of these data, and the research protocol of the study was approved by the ethics committee of medical care and research of the University of Occupational and Environmental Health, Kitakyushu, Japan.

\section{Patients}

Information from 3431 patients with HFS (ICD-10 codes: G 513) was collected using the DPC administrative database for 2010, 2011, 2012 and 2013. We excluded 524 patients who had not undergone MVD for HFS. Therefore, a total of 2907 patients were allocated for analysis. The 2907 patients were referred to 1074 DPC participating hospitals (83 academic and 991 community hospitals). The variables extracted from the DPC database were patient age and sex, residential area, discharge status, and the length of hospital stay. Furthermore, we categorized 47 prefectures into eight distinct districts, including the Hokkaido district (Hokkaido), Tohoku district (Aomori, Iwate, Miyagi, Akita, Yamagata, Fukushima), Kanto district (Ibaraki, Tochigi, Gunma, Saitama, Chiba, Tokyo, Kanagawa), Chubu district (Niigata, Toyama, Ishikawa, Fukui, Yamanashi, Nagano, Gifu, Shizuoka, Aichi), Kinki district (Mie, Shiga, Nara, Wakayama, Kyoto, Osaka, Hyogo), Chugoku district (Okayama, Hiroshima, Tottori, Shimane, Yamaguchi), Shikoku district (Kagawa, Tokushima, Ehime, Kochi), and Kyushu district (Fukuoka, Saga, Nagasaki, Oita, Kumamoto, Miyazaki, Kagoshima, Okinawa). We calculated the number of MVD treatments for the treatment of HFS conducted per year per 100,000 population in each district using the 2010 national census data.

\section{Results}

Analysis of the DPC data showed that from July 1, 2010, until March 31, 2013, MVD was used for the treatment of HFS in 2907 cases (men, 916 cases; women, 1991 cases) in DPC-participating facilities in Japan. The number of women treated with MVD was 2.2 times greater than the number of men treated with MVD. Overall, MVD for HFS was most frequently performed in patients in their 60 s, with the seventh decade of life the peak age for women undergoing MVD. However, many men underwent MVD treatment at an earlier age, with most aged between 40 and 59 years at the time of the procedure (Fig. 1). The numbers were lower for both male and female MVD patients aged in their 
$20 \mathrm{~s}$ and $80 \mathrm{~s}$. The female/male ratio of patients was 2.0 in total and 2.8 for patients in their 60 s and 70s (Table 1).

The Tokyo prefecture had the highest number of MVD procedures (494 cases/33 months), and the number of MVD treatments was larger in prefectures with a larger population (Fig. 2). In Japan, the numbers of MVD procedures performed for the treatment of HFS per 100,000 population/year in total and across the 8 districts were: 1) 0.83 overall; 2) 1.09 in the Hokkaido district; 3) 0.69 in the Tohoku district; 4) 0.85 in the Kanto district; 5) 0.74 in the Chubu district; 6) 0.76 in the Kinki district; 7) 0.91 in the Chugoku district; 8) 0.59 in the Shikoku district; and 9) 1.06 in the Kyushu district. The number of

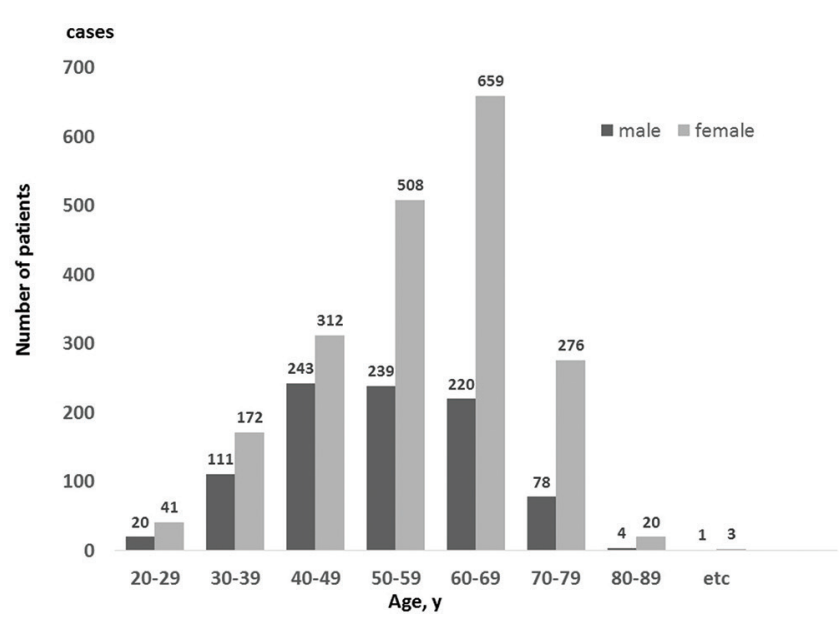

Fig. 1 The number of microvascular decompression (MVD) procedures for the treatment of hemifacial spasm during a 33-month period from July 2010 to March 2013 in each age group. There were 2907 cases, including 916 male cases and 1991 female cases. The number of female cases was 2.2 times higher than the number of male cases. MVD was most frequently performed in men in their 40s-50s but in women in their 60s.
MVD procedures performed in the Shikoku district was slightly more than half of the highest number observed in the Hokkaido district (Fig. 3). Regarding discharge outcomes, cure, improvement, or remission was achieved in 2874 of 2907 cases (98.9\%), and the mortality rate was $0.1 \%$. There was no apparent difference in discharge outcomes observed between men and women (Fig. 4). The mean length of hospital stay in patients undergoing MVD for HFS was 14.7 days (men, 14.5 days; women, 14.7 days) (Fig. 5).

\section{Discussion}

The use of MVD for the treatment of HFS has been investigated in single-center and multicenter studies; however, there have been no studies based on a national database such as the DPC database. ${ }^{15-17)}$ In this study, using DPC data, we analyzed the utilization of MVD for the treatment of HFS during the 33-month period from July 2010 to March 2013 in terms of the number of MVD procedures performed, regions where MVD was performed, length of hospital stay, and discharge outcomes. Not all medical institutions in Japan contribute to the DPC database and not all DPC data were submitted. In 2012, data were collected from 1057 institutions of 1774 DPC-participating hospitals, accounting for approximately $60 \%$ of all DPC data. However, certain neurosurgical procedures, such as MVD, are presumably performed primarily at acute care hospitals that typically contribute to the DPC database. In fact, in 2014, $88.1 \%$ of institutions belonging to the Japanese Society for Microvascular Decompression Surgery contributed to the DPC database. In consideration of these factors, including the specialized nature of the procedure and the proportion of DPC-participating institutions in the Japanese Society for Microvascular Decompression Surgery, the data obtained from this study should

Table 1. The number of male and female patients undergoing microvasculature decompression for the treatment of hemifacial spasm

\begin{tabular}{lcccc}
\hline $\begin{array}{l}\text { Age on } \\
\text { Enforced MVD }\end{array}$ & $\begin{array}{c}\text { Total Enforcement } \\
\text { Rate per } \\
\text { 100000/Year }\end{array}$ & $\begin{array}{c}\text { Male Enforcement } \\
\text { Rate per } \\
\text { 100000/Year }\end{array}$ & $\begin{array}{c}\text { Female } \\
\text { Enforcement Rate } \\
\text { per 100000/Year }\end{array}$ & $\begin{array}{c}\text { Total Enforcement } \\
\text { Rate per 100000/Year } \\
\text { Female: Male Ratio }\end{array}$ \\
\hline $20-29$ & 0.2 & 0.1 & 0.2 & $2.0: 1$ \\
$30-39$ & 0.6 & 0.4 & 0.7 & $1.8: 1$ \\
$40-49$ & 1.2 & 1.0 & 1.4 & $1.4: 1$ \\
$50-59$ & 1.7 & 1.1 & 2.2 & $2.0: 1$ \\
$60-69$ & 1.8 & 0.9 & 2.5 & $2.8: 1$ \\
$70-79$ & 1.0 & 0.5 & 1.4 & $2.8: 1$ \\
$80-89$ & 0.1 & 0.1 & 0.2 & $2.0: 1$ \\
Total & 1.0 & 0.7 & 1.4 & $2.0: 1$ \\
\hline
\end{tabular}




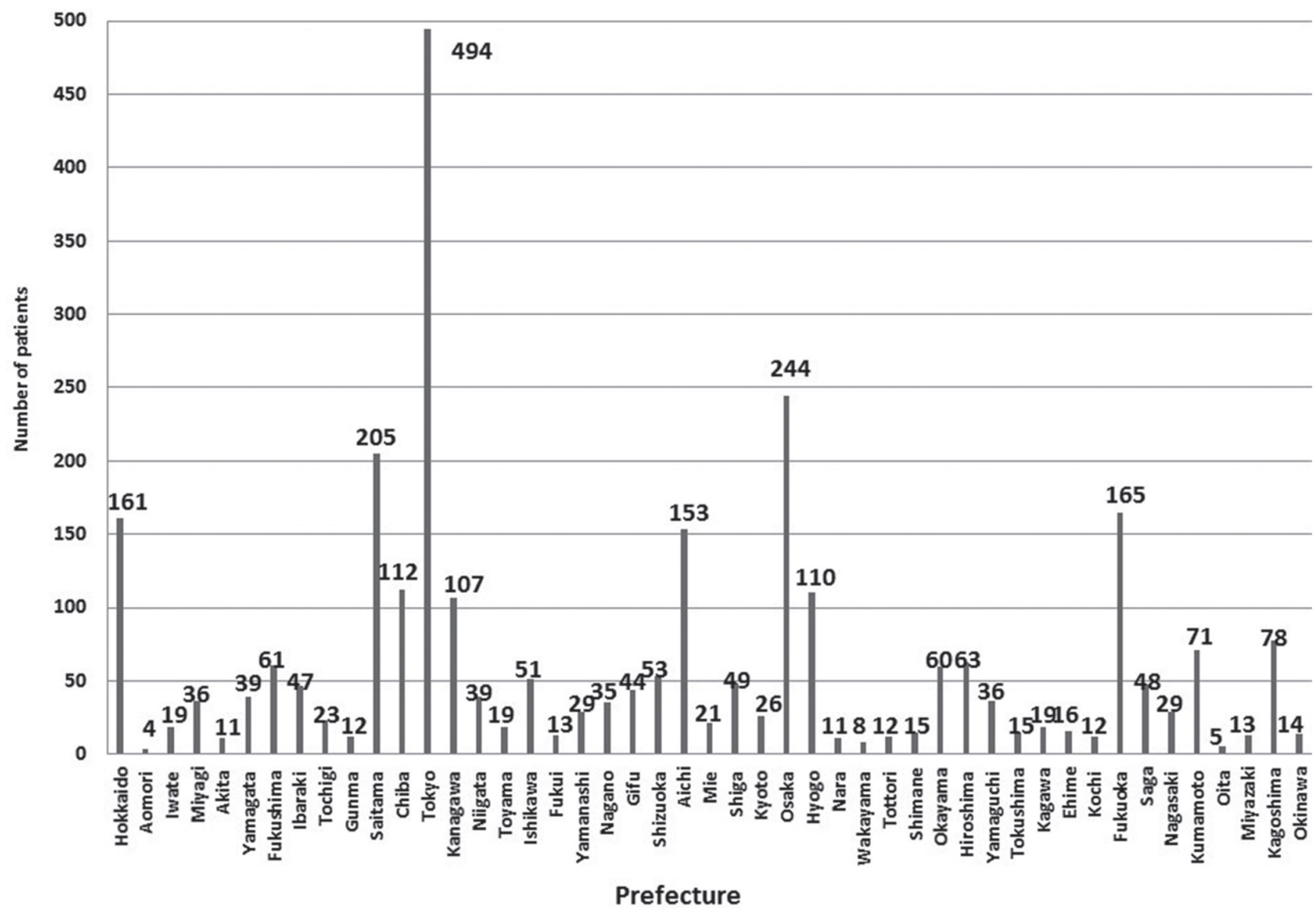

Fig. 2 The number of cases of microvascular decompression for the treatment of hemifacial spasm performed during a 33-month period from July 2010 to March 2013 in each prefecture. Tokyo had the highest number at 494 cases, and Osaka had 244 cases. MVD was more frequently performed in prefectures with a larger population.

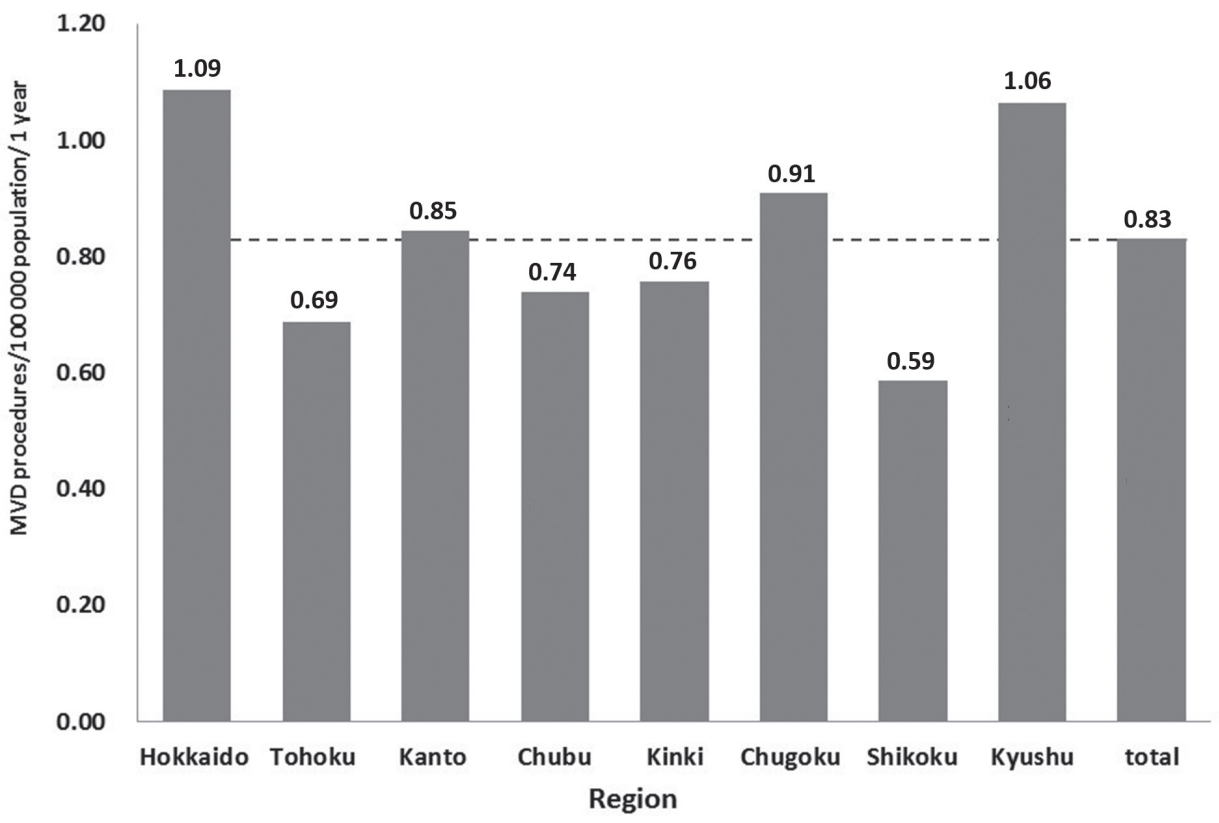

Fig. 3 The number of patients undergoing microvascular decompression for the treatment of hemifacial spasm per 100,000 population per year was compared between 8 districts. The 47 prefectures were divided into 8 distinct districts. Overall, in Japan, 0.83 patients/100,000 population/ year were treated. Among the districts, the Hokkaido district had the highest number (1.09 cases/100,000 population/year), whereas the Shikoku district had the lowest number $(0.59$ cases/100,000 population/year). 


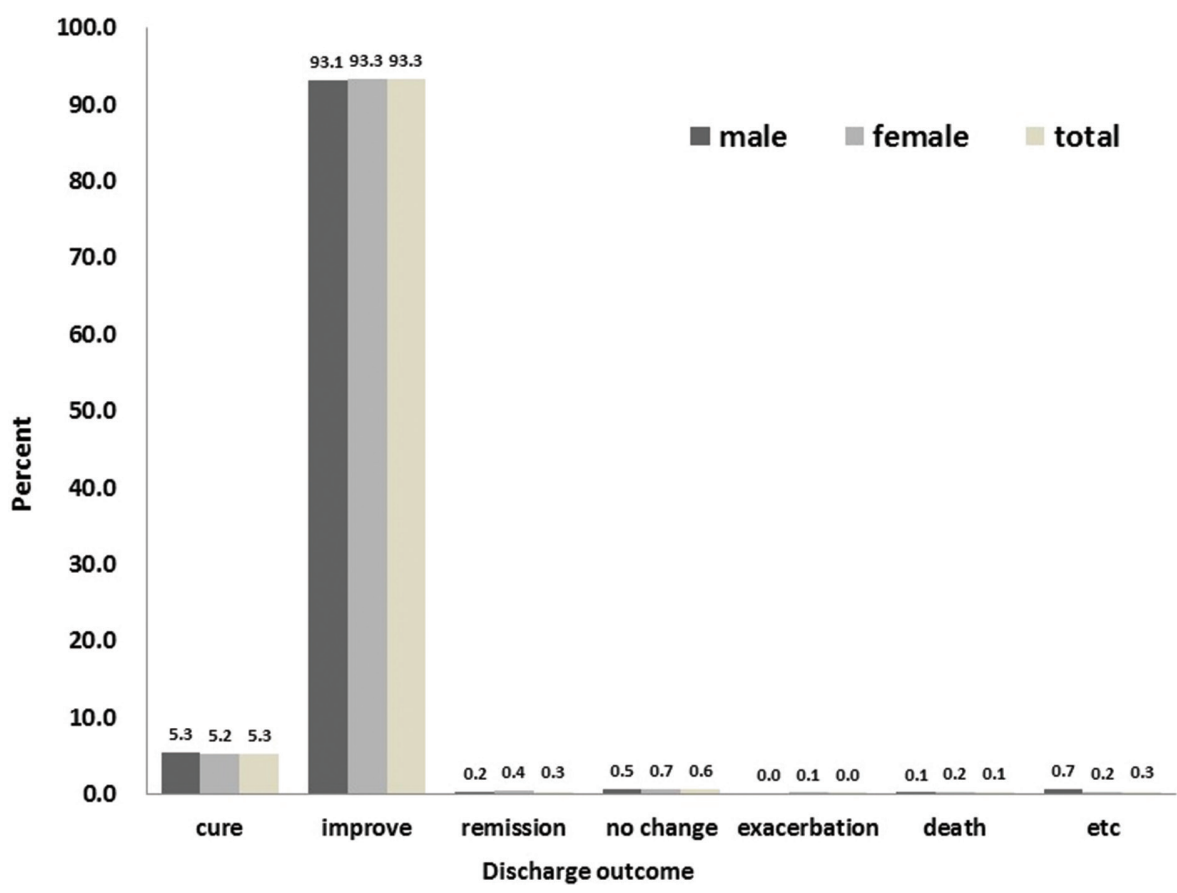

Length of hospital stay

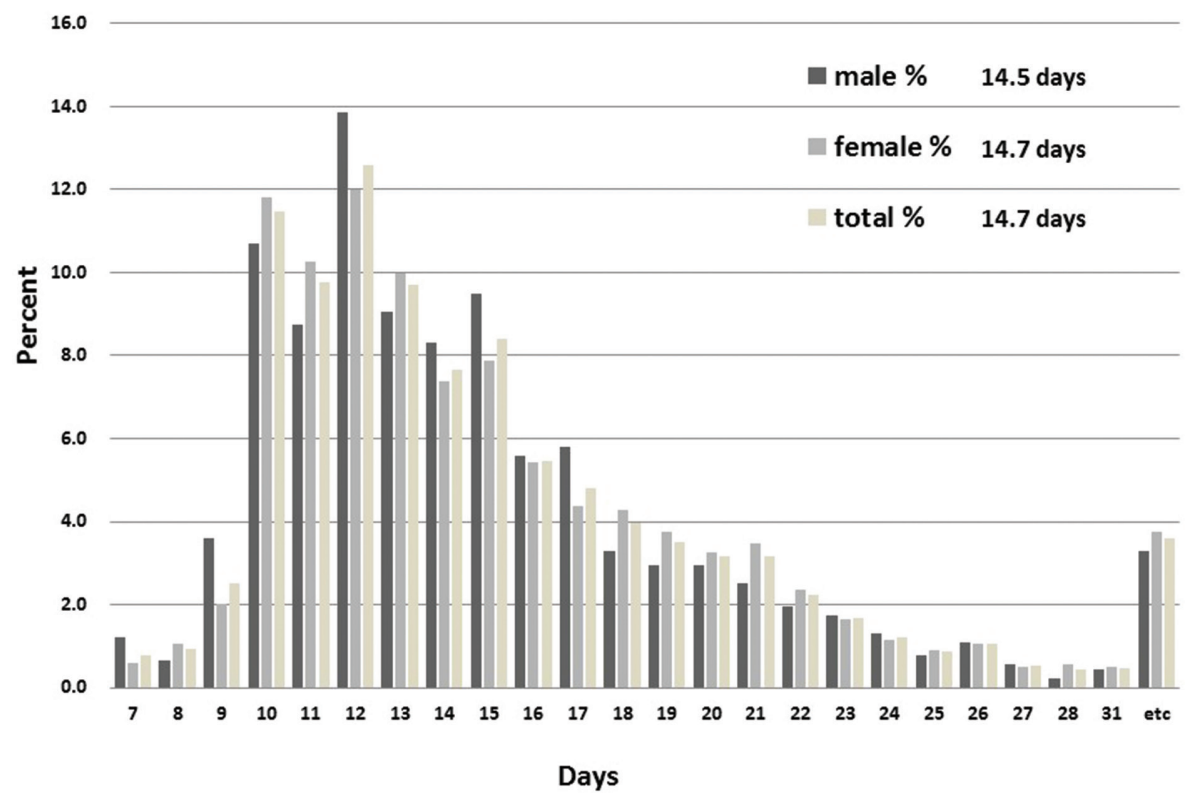

Fig. 4 Discharge outcomes in patients undergoing microvascular decompression for the treatment of hemifacial spasm. Outcomes were assessed as cure, improvement, remission, no change, exacerbation, death, and other conditions. Patients with cure, improvement, or remission accounted for $\mathbf{9 8 . 9} \%$ of all patients, and death occurred in $0.1 \%$ of patients.

Fig. 5 Length of hospital stays in patients undergoing microvascular decompression for the treatment of hemifacial spasm. Overall, the mean length of hospital stay was 14.7 days. No difference in the length of stay was observed between men and women.

accurately reflect trends in the use of MVD for the treatment of HFS in Japan.

According to the DPC data, MVD for HFS had been performed in Japan with a male-to-female ratio of $1: 2.2$. In the literature, the annual incidence rate of HFS is 0.78 (women, 0.81 ; men, 0.74) per 100,000 population, with almost no difference in the incidence rates between men and women. ${ }^{18-20)}$ However, the morbidity rate of HFS is $14.5 / 100,000$ population in women and 7.4/100,000 population in men, producing a sex ratio of $2: 1 .^{18-20)}$ Therefore, it is believed that the MVD male-to-female treatment ratio of $1: 2.2$ is linked to the differential morbidity rates for HFS in men and women.

According to our DPC study, MVD is most often performed in women between 50 and 69, whereas it is frequently performed in men between 40 and 59 years of age. Thus, the peak number of MVD procedures performed in men was at a younger age than in women. Because there are no differences between men and women in the age adjusted incidence rates, ${ }^{19)}$ this result suggests that men tend 
to undergo MVD treatment at a younger age than women in Japan.

Regarding the number of MVD procedures performed per 100,000 population/year in each district, the Shikoku district had the lowest number at 0.59 procedures, which was a little more than half of the highest number observed in the Hokkaido district (1.09 procedures/100,000 population/year). We believe that this difference can be attributed to a trend toward surgical treatment of HFS in the Hokkaido and Kyushu districts, as opposed to a trend toward conservative therapy in the Shikoku district. However, DPC data do not provide information on the status of botulinum toxin therapy administered at outpatient clinics, and this may affect the frequency of MVD utilization in the different districts. We do not believe that this difference in the use of MVD can be attributed to the differences in the morbidity rates in the different districts.

Regarding treatment outcomes at discharge, cure, improvement, or remission was achieved in $98.9 \%$ of all cases, and the mortality rate was $0.1 \%$. Compared to the results of other reports, these outcomes appear to be favorable..$^{7,15,18,21-23)}$ However, since the DPC system lacks strict criteria for assessing outcomes at discharge for patients undergoing MVD, the results may not be directly comparable to those obtained in other studies.

\section{Study Limitations}

This study had several limitations. First, the studied data does not cover the all DPC participatory hospitals. The data were obtained only from DPC-participating hospitals that contracted with the DPC research institute, not all medical institutions in Japan. In 2012, data were collected from 1057 institutions of 1774 DPC-participating hospitals, accounting for approximately $60 \%$ of all DPC data. For these reasons, the numbers of MVD executed in each area are only reference values, and are believed to be lower than the actual numbers. Therefore, data in non-participating hospitals should be analyzed in the future to confirm the current status of MVD for the treatment of HFS in Japan.

Second, DPC data are not useful for determining the incidence of complications in MVD, such as auditory disorders, dysphagia, and facial paralysis, because of the limitations of this database. Therefore, analysis of a disease-specific registry would be preferable.

Finally, the DPC data do not provide information on the long-term outcomes after MVD for the treatment of HFS because the DPC database only includes in-hospital data.

\section{Conclusion}

Because the DPC database is not a comprehensive registry, not all relevant cases can be analyzed in an investigative study using the DPC database, and detailed analysis is difficult to perform due to the limitations of this database. For example, it is not a disease-specific registry and does not include data on the treatment administered at outpatient clinics. However, analysis of the DPC data revealed trends in the performance of MVD for the treatment of HFS in Japan. The database also revealed largely favorable outcomes for the procedure. However, the DPC database is not sufficient to determine the efficacy of MVD for the treatment of HFS or the incidence of complications in Japan. Further studies on outcomes need to be conducted with other registries that contain data obtained by standardized assessment methods and that include long-term outcomes and postoperative complications.

\section{Acknowledgment}

Disclosure of funding: We have no sources of financial support to disclose.

\section{Conflicts of Interest Disclosure}

The authors declare that they have no competing interests.

\section{References}

1) Campos-Benitez M, Kaufmann AM: Neurovascular compression findings in hemifacial spasm. J Neurosurg 109: 416-420, 2008

2) Jo KW, Kong DS, Park K: Microvascular decompression for hemifacial spasm: long-term outcome and prognostic factors, with emphasis on delayed cure. Neurosurg Rev 36: 297-301, 2013

3) Li Y, Zheng X, Hua X, Ying T, Zhong J, Zhang W, Li S: Surgical treatment of hemifacial spasm with zone-4 offending vessel. Acta Neurochir (Wien) 155: 849-853, 2013

4) Gardner JW, Sava GA: Hemifacial spasm-a reversible pathophysiologic state. J Neurosurg 19: 240-247, 1962

5) Jannetta PJ: The cause of hemifacial spasm: definitive microsurgical treatment at the brainstem in 31 patients. Trans Sect Otolaryngol Am Acad Ophthalmol Otolaryngol 80: 319-322, 1975

6) Jannetta PJ, McLaughlin MR, Casey KF: Technique of microvascular decompression. Technical note. Neurosurg Focus 18: 1-5, 2005

7) Samii M, Günther T, Iaconetta G, Muehling M, Vorkapic P, Samii A: Microvascular decompression to treat hemifacial spasm: long-term results for a consecutive 
series of 143 patients. Neurosurgery 50: 712-718, 2002

8) Elston JS: The management of blepharospasm and hemifacial spasm. J Neurol 239: 5-8, 1992

9) Ainsworth JR, Kraft SP: Long-term changes in duration of relief with botulinum toxin treatment of essential blepharospasm and hemifacial spasm. Ophthalmology 102: 2036-2040, 1995

10) Murata A, Mayumi T, Muramatsu K, Ohtani M, Matsuda S: Effect of dementia on outcomes of elderly patients with hemorrhagic peptic ulcer disease based on a national administrative database. Aging Clin Exp Res 27: 717-725, 2015

11) Murata A, Muramatsu K, Ichimiya $Y$, Kubo T, Fujino Y, Matsuda S: Endoscopic submucosal dissection for gastric cancer in elderly Japanese patients: an observational study of financial costs of treatment based on a national administrative database. J Dig Dis 15: 62-70, 2014

12) Murata A, Okamoto K, Mayumi T, Maramatsu K, Matsuda S: Age-related differences in outcomes and etiologies of acute abdominal pain based on a national administrative database. Tohoku J Exp Med 233: 9-15, 2014

13) Murata A, Okamoto K, Muramatsu K, Matsuda S: Endoscopic submucosal dissection for gastric cancer: the influence of hospital volume on complications and length of stay. Surg Endosc 28, 1298-1306, 2014

14) Murata A, Okamoto K, Muramatsu K, Kubo T, Fujino Y, Matsuda S: Effects of additional laparoscopic cholecystectomy on outcomes of laparoscopic gastrectomy in patients with gastric cancer based on a national administrative database. J Surg Res 186: 157-163, 2014

15) Huh R, Han IB, Moon JY, Chang JW, Chung SS: Microvascular decompression for hemifacial spasm: analyses of operative complications in 1582 consecutive patients. Surg Neurol 69: 153-157, 2008

16) Lee MH, Jee TK, Lee JA, Park K: Postoperative complications of microvascular decompression for hemifacial spasm: lessons from experience of 2040 cases. Neurosurg Rev 39: 151-158, 2016

17) Kalkanis SN, Eskandar EN, Carter BS, Barker FG: Microvascular decompression surgery in the United States, 1996 to 2000: mortality rates, morbidity rates, and the effects of hospital and surgeon volumes. Neurosurgery 52: 1251-1261, 2003

18) Abbruzzese G, Berardelli A, Defazio G: Hemifacial spasm. Handbook of Clinical Neurology 100: 675-680, 2011

19) Auger RG, Whisnant JP: Hemifacial spasm in Rochester and Olmsted County, Minnesota, 1960 to 1984. Archiv Neurol 47: 1233-1234, 1990

20) Nilsen B, Le KD, Dietrichs E: Prevalence of hemifacial spasm in Oslo, Norway. Neurology 63: 1532-1533, 2004

21) Illingworth RD, Porter DG, Jakubowski J: Hemifacial spasm: a prospective long-term follow up of 83 cases treated by microvascular decompression at two neurosurgical centres in the United Kingdom. J Neurol Neurosurg Psychiatry 60: 72-77, 1996

22) Chang WS, Chung JC, Kim JP, Chung SS, Chang JW: Delayed recurrence of hemifacial spasm after successful microvascular decompression: follow-up results at least 5 years after surgery. Acta Neurochir (Wien) 154: 1613-1619, 2012

23) Sekula RF, Frederickson AM, Arnone GD, Quigley MR, Hallett M: Microvascular decompression for hemifacial spasm in patients $>65$ years of age: an analysis of outcomes and complications. Muscle $\mathcal{E}$ Nerve 48: 770-776, 2013

Address reprint requests to: Yoshifumi Mizobuchi, MD, PhD, Department of Neurosurgery, Institute of Health Biosciences, The University of Tokushima Graduate School, 3-18-15 Kuramoto-cho, Tokushima, Tokushima 770-8503, Japan e-mail: mizo@yj8.so-net.ne.jp 